\title{
Towards an establishment of a rating curve for suspended sediment transport by means of ADCP measurements
}

\author{
Nils Rüther, ${ }^{1, *}$, Rui Aleixo ${ }^{2}$, Massimo Guerrero ${ }^{2}$, Sigurd Sørås ${ }^{3}$ and Siri Stokseth ${ }^{4}$ \\ ${ }^{1}$ NTNU, Department of Civil and Environmental Engineering, S.P. Andersensveg 5, 7022 Trondheim, \\ Norway \\ ${ }^{2}$ University of Bologna, DICAM, via Umberto Terracini 28, 40131 Bologna, Italia \\ ${ }^{3}$ Multiconsult ASA, Nedre Skøyen vei 2, 0276 Oslo \\ ${ }^{4}$ Statkraft As, Lilleakerveien 6, Postboks 200 Lilleaker, 0216 Oslo, Norway
}

\begin{abstract}
This study presents a series of acoustic current Doppler profiler (ADCP) measurements, which are converted into suspended load concentrations (SSC). Depending on the particle size distribution (PSD), the sound backscatter and its attenuation are used to determine a theoretical minimal and physical upper limit of the SSC. The SSC are displayed over time and compared with the evolution of the water level during the same time interval. The present study shows that it is possible to have continuous measurements of SSC and water level with a horizontally installed two beam equipped $\mathrm{ADCP}$ and discusses the potential of such a continuing times series measurement for application in hydraulic engineering and research. However, the presented data set shows also that the calibration and the filtering of the raw data has to be improved and that further investigations are needed. After data verification, it will be possible to calculate the yearly sediment load and investigate physical behaviour of SSC concentration as a function of water level and discharge.
\end{abstract}

\section{Introduction}

The global development of hydropower is an important step towards a low-carbon society. However, in regions with large hydropower potential such as South-East Europe, Asia, Africa and South America sediments and sedimentation processes are core challenges that need to be addressed and amended to ensure sustainable hydropower development. Sedimentation and erosion processes have proven to be technically, economically and socio-ecologically, challenging. Additionally, climate change is projected to cause increased sediment catchment yield, affecting the operation and maintenance of the hydropower plants and hence energy production. In order to make the energy production and the use of the reservoir sustainable, the design and operation of hydropower plants have to be based on high quality data series of sediment transport in space and time in the adjacent rivers.

As it is per today, it takes relatively much time to take physical samples from the river and then investigate the samples in the laboratory for the analysis of SSC and PSD. In addition,

\footnotetext{
* Corresponding author: nils.ruther@ntnu.no
} 
it is difficult or sometimes impossible to measure at higher discharge due to physical limitations of the measurement devices as well as due to health and safety regulations. Unfortunately, these high discharges are responsible for high loads of sediment. Consequently, the calculated total load over the year is usually strongly underestimated. The aforementioned shortcomings, being cost intensive and unreliable, make it necessary to develop and verify cost-effective methods for sediment transport measurements. An additional use for such detailed measurement of SSC in rivers and reservoirs can be the operation, calibration and or verification of numerical models. Many studies show that with a detailed time series as upstream boundary condition, the results can be improved majorly, e.g. $[1,2]$

Therefore, this study is presenting the application of an acoustics based method to quantify suspended transport load in a reach of the Devoll river upstream of the Banja hydro power plant in Albania. Many studies have been dealing with the estimation of SSC from acoustic backscatter signal, e.g. $[3,4,5,6]$. The present study uses a method based on the Guerrero \& Di Federico [7], in which the attenuation and backscatter signal is related to SSC as well as to PSD and allows obtaining a data series of high time resolution.

\section{Experimental site and setup}

The experimental site is located in the Devoll river in central Albania. The site, indicated with white circle in Figure 1, is located upstream of the hydro power plant of Banja (white square in Figure 1). The river downstream of the measurement site is characterised by being strongly braided with a wide open cross section area. The river carries over $80 \%$ of the total sediment load as suspended load. The river Tomorrica coming in flow direction from the left side triturating to Devoll, carrying up to $50 \%$ bed load. Upstream of the Kokel bridge, the river is characterised by a narrow gorge being partly $100 \mathrm{~m}$ incised in the landscape.

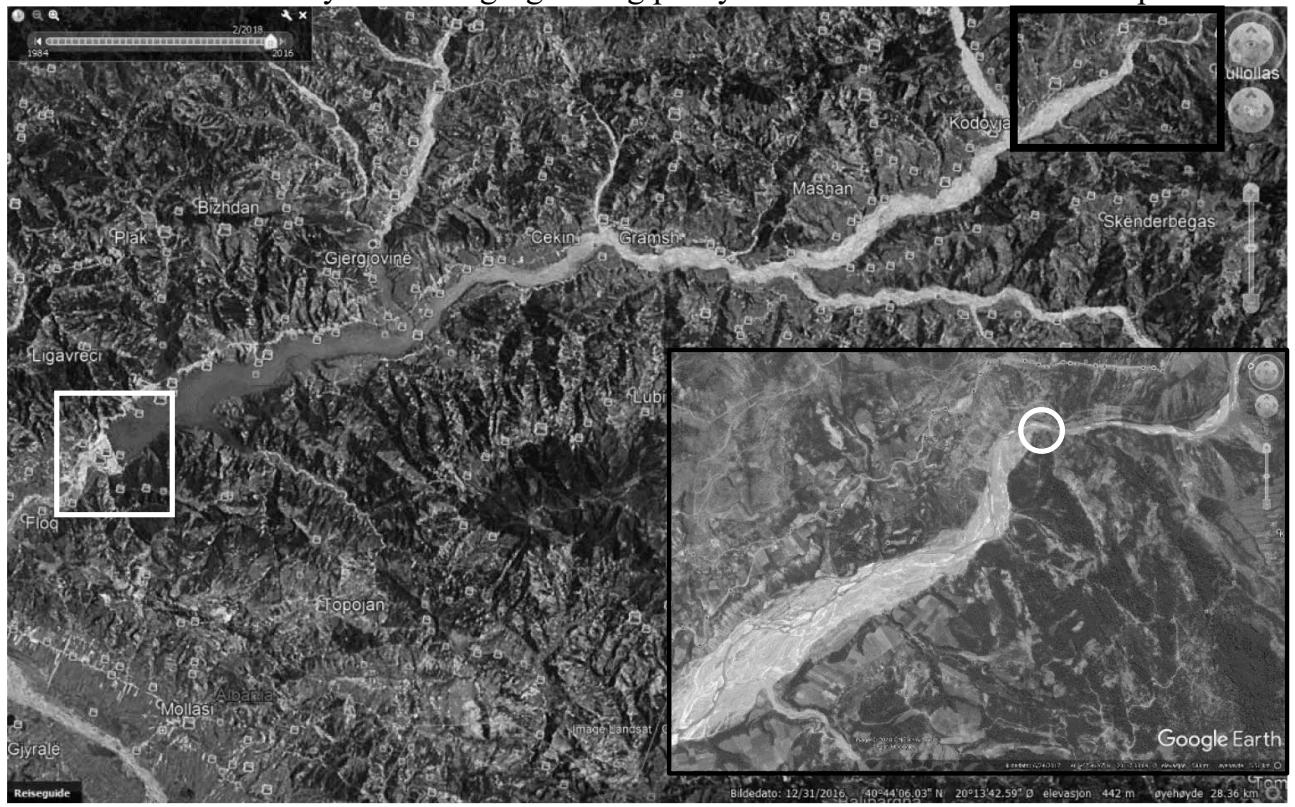

Figure 1: Overview of the location of the measurement site. (Ref: from Google earth)

In Figure 1 in the upmost right corner, Kokel bridge, white circle, and its downstream area is displayed. Looking at the bottom right frame, one can see the braided character of the river and the gorge type valley upstream Kokel bridge. The sediment transport research station in 
Kokel was established in October 2015. At this station, two horizontal ADCPs were installed to permanently log the acoustic backscatter and attenuation signal reflecting from the particles in the water column. In addition, water level is measured by the built-in ultra-sonic sensors pointing vertically up to the water surface. The measurement series starts from October 2015 and will be acquiring data until June 2018. In this study, data from February to July 2016 is presented. The method for the determination of the SSC is described mainly in and it is not topic of the paper. The application of the mentioned method to the herein discussed data is presented in .

Figure 2 shows the experimental setup in the field. In Figure 2a one can see the measurement cross section seen from the right side of the river. The cross section is situated in a mild bend. Two horizontal ADCPs mounted on a vertical platform at the outside of the river bend, to measure in the cross section, pointing towards the inner part of the bend. Figure $2 b$ shows the view towards upstream from the measurement cross section. The profile of the cross section is illustrated Figure 2c. The two horizontal ADCPs are mounted at a height of $0.8 \mathrm{~m}$ above the zero level. Consequently, the lowest measurement condition is determined for a water level of $1.0 \mathrm{~m}$ in the local coordinate system. During the 2.5 years of measurement, several flood events have been recorded, e.g. the one of $22^{\text {nd }}$ of October in 2015 with a maximum level of $2.45 \mathrm{~m}$. The ADCP data is aquired with a frequency of $0.5 \mathrm{~Hz}$.
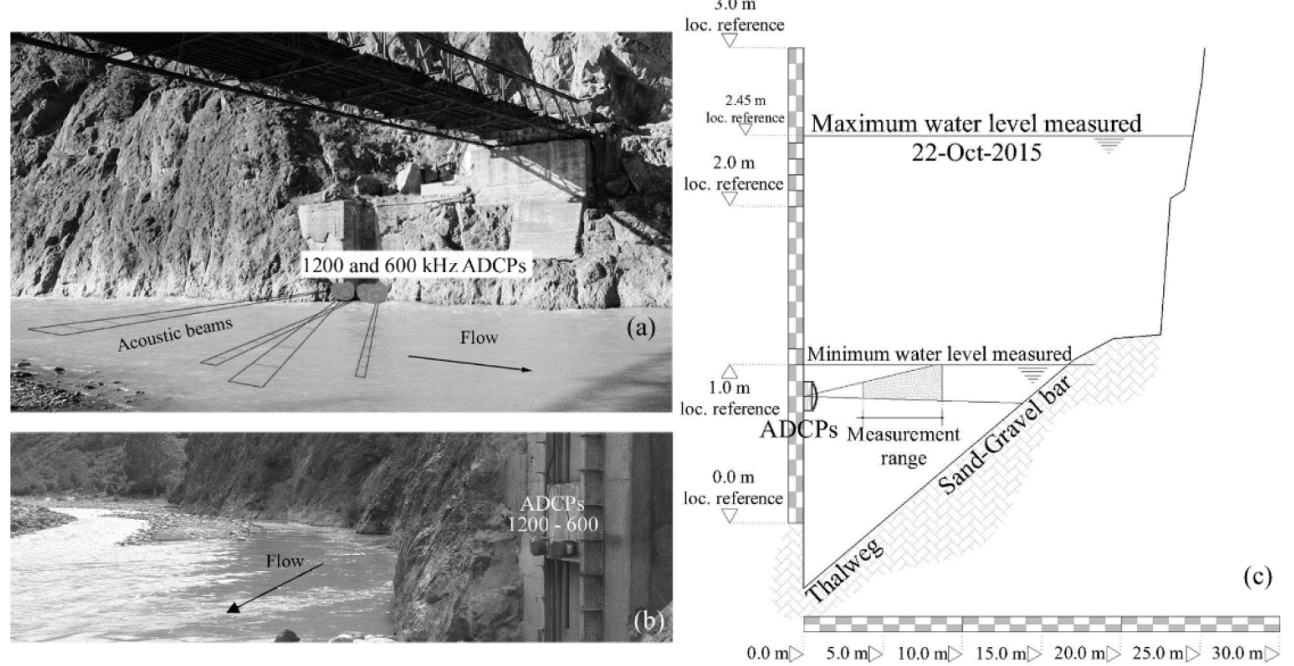

$0.0 \mathrm{~m}>5.0 \mathrm{~m} \gg 10.0 \mathrm{~m} \gg 15.0 \mathrm{~m} \gg 20.0 \mathrm{~m}>25.0 \mathrm{~m} \triangleright>30.0 \mathrm{~m}>$

Figure 2 : Picture and sketch of the experimental setup at the sediment transport research station in Kokel, which is distorted in the vertical scale for the sake of clarity [8]

\section{Suspended load measurements and data processing}

\subsection{Physical, acoustic and optical point sampling}

Point sampling have been carried out in several ways. Physical sampling with a bottle sampler from the side of the river at low water level condition has been conducted on a regular basis throughout the year. In addition, isokinetic point integrated sampling with the P-72 by USGS was performed during different flow conditions. The samples have been analysed for SSC and PSD. Sparse samples have been taken with the LISST SL by Sequoia. This device is a laser diffraction device which measures SSC and PSD with $1 \mathrm{~Hz}$ in real time. Almost no post processing for this data is needed for the LISST-SL measurements. Some samples were taken with the acoustic backscatter sensor (ABS), also provided by Sequoia. The device is 
calibrated for a PSD with a d50 as inferior index in the range of 30-300 microns. If the prevailing PSD deviates from that, the data has to be calibrated. In addition to the isokinetic methods, an ISCO sampler was installed to ensure to also collect data during high discharges. The controller is programed so that the samplers starts collecting samples as soon as a threshold water level is exceeded. The collected samples were analysed for SSC and PSD with the so-called LISST-Portable, which is also based on the optical laser diffraction method.
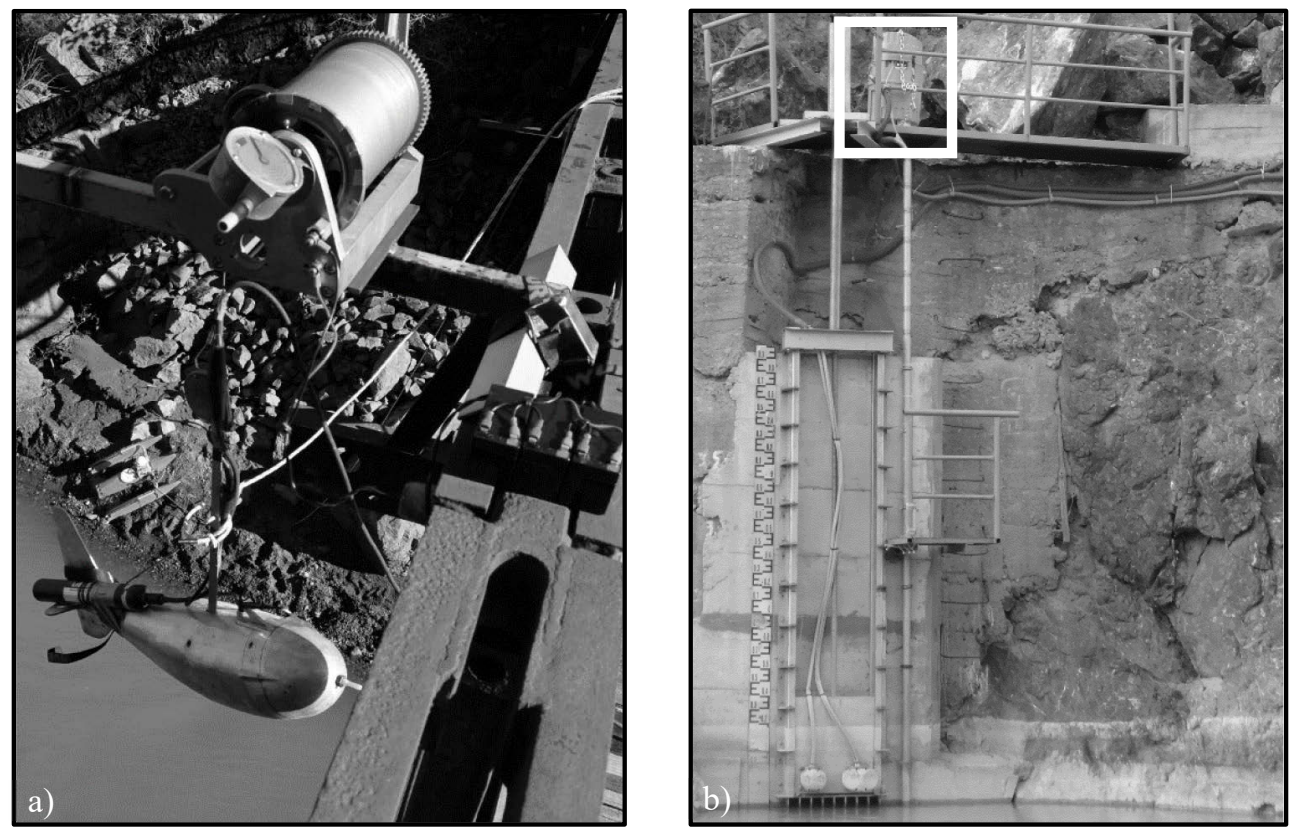

Figure 3: Physical sampling with isokinetic point-integrated samplers- US P-72 equipped with a Sequoia Acoustic backscatter sensor (ABS) by Sequoia (a). Point sampler by ISCO (b)

\subsection{Acoustic profiling method}

The acoustic method here used was put forward by [7] and it works based on the ratio between acoustic attenuation and backscatter (ABR) and concentration by means of a convenient function. To determine some of the parameters of this function, physical properties of the sediments (mean diameter, standard-deviation, skewness, i.e., the particle size distribution (PSD)) and SSC have to be known in order to calibrate and validate the method. A critical aspect of this method for the attenuation calculation is the determination of the echo's profiles slopes. This method application can be seen in [9]. The method's results from 1st February to 31 st of July 2016 can be seen in Figure 4. The displayed result for the SSC is based on averaged value of the echo profile over 8 minutes. Figure 4 shows the water level evaluation on the left $\mathrm{y}$-axis and the resulting SSC on the right y-axis, both over time. Looking at Figure 4 it can be stated that the SSC are generally following the change in water level. A rise in the water level leads to a rise in SSC and consequently the SSC falls when the levels drops. However, looking more closely at Figure 4, it can be seen that some peaks of SSC are shifted in time, comparing the moment of the peak of SSC with the one of the discharge. This observation leads to the assumption that the phenomenon of hysteresis can be observed over time. This assumption can first be verified when the discharge data will be post processed and the SSC data will be verified in the near future once more physical samples are measured and post processed. 
In general, for the above mentioned period, the water level decreases over the displayed time. This can be explained by the general reduction of water in the river during spring time. The hydrology is characterized by a rain-influenced autumn with many flood events of different return intervals, during October and December. In spring, the water levels are not only influenced by the melting water, but also by small rain events.

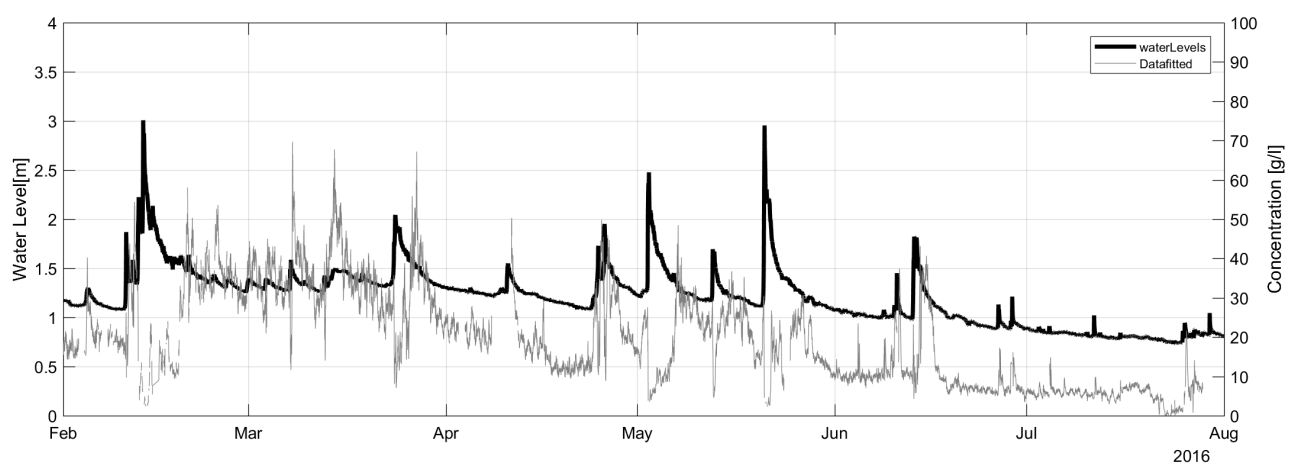

Figure 4 : Measured water level and SSC between 01.02. and 31.07.2016. Water level on the right yaxis, SSC on left y-axis

In general, the water level decreases towards the summer and so does the SSC. Some small events trigger an increase in the SSC and this can be observed in the data. Consequently this is also expected in the large events of rain. However, this cannot be fully confirmed with the measured data converted to SSC. Some of the larger water level rises are followed by an abrupt fall of the SSC. In addition, the absolute values of the resulting concentration, which are displayed in here, may vary significantly after the full calibration has been conducted. . Physical, optical and acoustic point samples, shows that the concentration occurring in this river are between 0.5 and $15 \mathrm{~g} / 1$ at maximum. This is mostly due to the fact that the calibration of the method needs to be based on more samples with different concentration values.

\section{Results and discussion}

Figure 5 shows the resulting data for a shorter time period, from February $23^{\text {rd }}$ to March $21^{\text {st }}$, 2016. From this figure, the true potential value of such a data series becomes clearer to the reader. The detailed measurement of water level and corresponding SSC can be analyzed much more detailed. In general, the resulting SSC follows the water level.

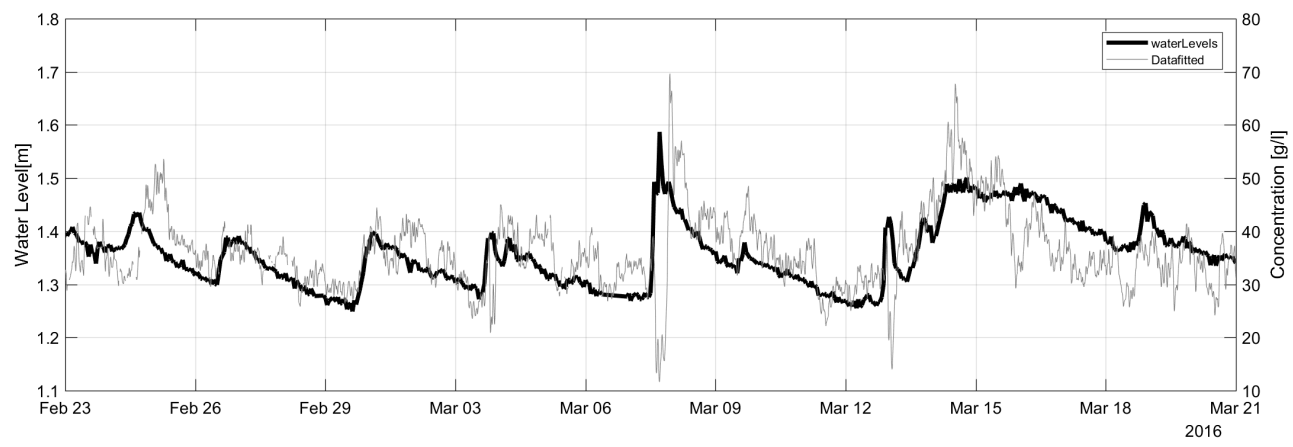

Figure 5 : Measured water SSC and SSC for the time period from 23.02.- 21.03.2016. Water level on the right y-axis, SSC on left y-axis 
However, during the fall of the water level, several peaks of SSC appear. This leads to the assumption of sudden river bank or slope collapses, delivering sediments to the river. In addition, it can be observed that there is time lag between several corresponding peaks which leads again to the hypothesis of prevailing hysteresis effects.

Figure 6 shows a further time period of 30 days: from April $20^{\text {th }}$ to May $20^{\text {th }}, 2016$. Here one can observe at least one quite sharp and abrupt rise in the water level just after May 2 . Unfortunately, the SSC for the corresponding time does not behave in a physical manner. Once the water level rises, the SSC drops in the same way, the water level rises

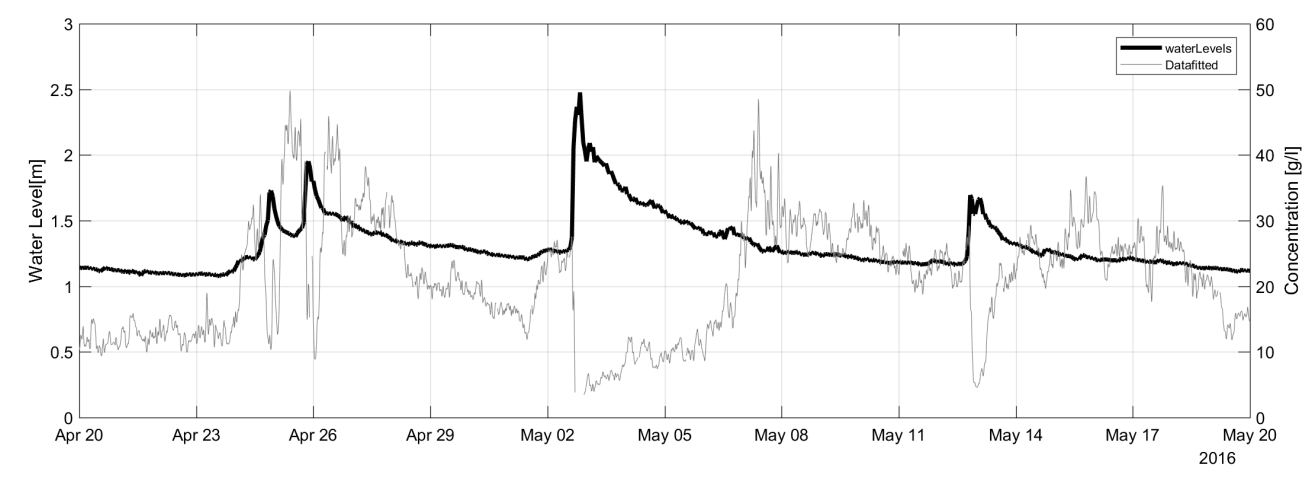

Figure 6 : Measured water SSC and SSC for the time period from April $20^{\text {th }} .-$ may $10^{\text {th }}, 2016$. Water level on the right $\mathrm{y}$-axis, SSC on left $\mathrm{y}$-axis

In Figure 6 just after May 2, the water level rises by $1.25 \mathrm{~m}$ in only $8 \mathrm{hrs}$. Within this study it remains unclear what is the reason for this behavior. Further effort in post processing the data will reveal in how far the echo profiles can be filtered in order to get to satisfactory results for such flow conditions. A physical explanation for this behavior might be the fact that due to very extreme conditions in terms of water level, flow velocity and turbulence, the emitted and received pulses uncorrelate and therefore lowering the quality of ADCP data.

\section{Conclusion outlook}

This study presents the preliminary results of a measurement campaign performed within the SediPASS project. One goal of this the project was to determine with a more reliable and more cost effective method to measure SSC in a river. To achieve this goal, two horizontal ADCPs have been installed at the sediment transport research station at Kokel bridge in the river Devoll in Albania. In addition to the measurement with the ADCPs, several conventional methods for SSC measurements were applied in order to obtain calibration and verification data.

The herein presented preliminary data series spans the time of 6 month in 2016. In February and July several small and medium flow rises and consequent rises in SSC could be observed. The data analysis showed that it is in principle possible to analyze physical behavior of water level and SSC interaction. However, the data has still to be carefully calibrated against physical samples taken at low and high prevailing SSC, together with the corresponding PSD. The presented data is calibrated using samples for low values, only. That is one of the reasons why the absolute values of the presented data must be treated with care. In addition, the analysis showed that the quality of the shape of the echo profiles varies a lot over time. It is observed that the echos change drastically with water level, and with the flow velocity, show a significant and sudden rise. During this period, the presented method resulted in incorrect lower bound values in the form of a drop in the SSC which is not physical. 
Further work will include the full calibration with all necessary physical samples and a more thorough and individual processing of the recorded echo profiles in order to predict realistic values for the SSC. In addition, the full series of discharge values to the according SSC values will be available in order to plot the full rating curve. Then it will also be possible to investigate the above mentioned hysteresis effects.

The authors acknowledge the project SediPASS funded by the Research Council of Norway with the project number 244104 as well as the INFRASAFE project funded by the Emilia Romagna Region in Italy by the European Regional Development Fund (POR-FESR), in the framework of the cohesion policy .

\section{References}

1. N. Rüther, M. Guerrero, and A. Lamberti, Modelling alluvial channel dynamics in a river reach dominated by alternate bars in 5th International Conference on Fluvial Hydraulics, River Flow 2010, A. Dittrich, Editor. 2010, Federal Waterways Engineering and Research Institute: Braunschweig, Germany. .

2. P. Zinke, N.R.B. Olsen, and J. Bogen, Geomorphology. 129(3-4): p. 320, (2011).

3. P.D. Thorne and R. Meral, Continental Shelf Research. 28(2): p. 309, (2008).

4. S.A. Moore, et al., Continental Shelf Research. 46: p. 50, (2012).

5. D.M. Hanes, Continental Shelf Research. 46: p. 64, (2012).

6. M. Guerrero, et al., Water. 8(1)(2016).

7. M. Guerrero and V. Di Federico, Advances in Water Resources. 113: p. 167, (2018).

8. M. Guerrero, et al. in River Sedimentation - Proceedings of the 13th International Symposium on River Sedimentation, ISRS 2016. 2017.

9. R. Aleixo, M. Guerrero, N. Rüther, S. Stokseth, RiverFlow 2018, under review. 